\title{
Anaesthesia for Liver Transplantation: An Update
}

\author{
Lavinia Nicoleta Brezeanu ${ }^{1 *}$, Radu Constantin Brezeanu², Mircea Diculescu ${ }^{1,3}$, Gabriela \\ $\operatorname{Droc}^{1,3}$ \\ ${ }^{1}$ Fundeni Clinical Institute, Bucharest, Romania \\ 2 "Bagdasar-Arseni" Emergency Hospital, Bucharest, Romania \\ 3 "Carol Davila" University of Medicine and Pharmacy, Bucharest, Romania
}

\begin{abstract}
Liver transplantation (LT) is a challenging surgery performed on patients with complex physiology profiles, complicated by multi-system dysfunction. It represents the treatment of choice for end-stage liver disease. The procedure is performed under general anaesthesia, and a successful procedure requires an excellent understanding of the pathophysiology of liver failure and its implications. Despite advances in knowledge and technical skills and innovations in immunosuppression, the anaesthetic management for LT can be complicated and represent a real challenge. Monitoring devices offer crucial information for the successful management of patients. Hemodynamic instability is typical during surgery, requiring sophisticated invasive monitoring. Arterial pulse contour analysis and thermo-dilution techniques (PiCCO), rotational thromboelastometry (RO-TEM), transcranial doppler (TCD), trans-oesophageal echocardiography (TEE) and bispectral index (BIS) have been proven to be reliable monitoring techniques playing a significant role in decision making. Anaesthetic management is specific according to the three critical phases of surgery: pre-anhepatic, anhepatic and neo-hepatic phase. Surgical techniques such as total or partial clamping of the inferior vena cava (IVC), use of venovenous bypass (VVBP) or portocaval shunts have a significant impact on cardiovascular stability. Post reperfusion syndrome (PRS) is a significant event and can lead to arrhythmias and even cardiac arrest.
\end{abstract}

Keywords: liver transplantation, haemodynamic monitoring, post-reperfusion syndrome

Received: 25 August 2019 / Accepted: 30 January 2020

\section{INTRODUCTION}

Liver transplantation (LT) is the treatment of choice for end-stage liver disease regardless of its aetiology. Ever since the first transplant interventions in the 1960s, mortality rates after LT have significantly improved and have led to an increase in the number of successful procedures and improved outcomes.

Significant challenges remain for the transplant team as the procedure is performed on high-risk patients with impaired cardiovascular, pulmonary, renal and coagulation systems. Recent publications have indicated that transplant candidates are older, sicker and with multiple associate co-morbidities and organ dysfunctions compared to those treated in the past. Adequate perioperative care is essential for a prompt graft function which will improve organ system recovery and recipient's quality of life [1].

Though there is a potential worldwide liver graft shortage, the expansion of the donor pool using mar- ginal donors and increasing donor age has resulted, never the less, in reduced waiting list mortality [2]. A successful LT requires teams with a particular set of skills and competences, including a complete and detailed understanding of the multi-organic pathophysiology of liver failure and its implications and management during the three stages of surgery.

There have been many innovations, updates and procedural changes in the anaesthetic management of patients during this time. This article gives an overview of the current literature regarding anaesthetic management during liver transplantation and its singularities during the three stages of surgery.

\section{INDICATIONS FOR LIVER TRANSPLANTA-} TION

The indications for LT in patients with acute and chronic liver failure should be assessed independent of the aetiology and are listed in Table 1 [3]. 
The US and European countries have been using the Model for End-Stage Liver Disease (MELD) score for organ allocation since 2007. This is a grading system from 6 to 40 points, which depends on four laboratory variables: serum creatinine, serum bilirubin, the international normalised ratio (INR) and serum sodium and evaluates the severity of chronic liver disease for patients older than twelve years of age. The aetiology of liver disease has been shown to be inefficient in predicting patient mortality. The UK Model for End-Stage Liver Disease (UKELD) score is used in recipient selection and prioritisation in the UK.
Certain medical conditions defined as "standard MELD exceptions" are eligible for an upgrade in MELD points and require periodic reassessment [4].

They include: hepatocellular carcinoma (HCC), hepatopulmonary syndrome, portopulmonary hypertension, familial amyloid polyneuropathy, primary hyperoxaluria, cystic fibrosis, hilar cholangiocarcinoma, hepatic artery thrombosis (within 14 days after LT but not meeting criteria for status $1 \mathrm{~A}$ ).

Another notable exception from the MELD prioritisation system is acute liver failure patients; they are UNOS status $1 \mathrm{~A}$ or $1 \mathrm{~B}$, and the severity and priority for LT are made according to King's College Criteria [5].

\section{Table 1. Indications for liver transplantation}

\section{Class}

Non-cholestatic liver disease

\section{Disease}

HepatitisB

HepatitisC

HepatitisD

HepatitisA

Alcoholic liver disease

Autoimmune hepatitis

Cryptogenic cirrhosis

Non-alcoholic steatohepatitis

Other

Cholestatic liver disease

Primary biliary cirrhosis

Secondary biliary cirrhosis (Caroli disease, choledochal cyst)

Primary sclerosing cholangitis

Other

Malignant disease

Hepatocellular carcinoma

Cholangiocarcinoma

Hepatoblastoma

Other

Extrahepatic biliary atresia or hypoplasia

Alagille syndrome

Other

Metabolic diseases

Hepatic vein thrombosis

Acute hepatic failure

Alpha-1 antitrypsin deficiency

Crigler-Najjar disease, Type I

Byler's disease

Glycogen storage disease, Type I

Wilson's disease

Hemochromatosis

Tyrosinemia

Wolman's disease

Familial amyloidotic polyneuropathy

Primary hyperoxaluria type 1

Other

Budd-Chiar
Hepatitis
Drugs

Unknown aetiology

Re-transplantation
Primary non-function

Hepatic artery thrombosis

Acute/chronic rejection 
The Milan criteria is used in selecting patients with primary liver tumours who may benefit from LT. Patients with a single tumour of $5 \mathrm{~cm}$ or less, or up to 2-3 tumours between $1-3 \mathrm{cms}$ in diameter, are considered to qualify for LT [6].

\section{ANAESTHETIC MONITORING}

\section{Stages of surgery}

LT is considered a complex procedure and is characterised by haemodynamic instability during the three phases of surgery:

Pre-anhepatic phase: from skin incision to clamping of the inferior vena cava (IVC), portal vein and hepatic artery; liver is being dissected, and significant bleeding can occur

Anhepatic phase: from the moment the hepatic venous inflow is being clamped up to graft reperfusion; the IVC is clamped causing a decrease in cardiac output $(\mathrm{CO})$

Neo-hepatic phase: it begins from the moment of liver reperfusion; resumption of flow in the portal vein and IVC; complicated by post-reperfusion syndrome (PRS) or bleeding from vascular anastomosis (IVC, hepatic artery or portal vein)

\section{Patient monitoring}

Anaesthetic management for liver transplant include:

Standard monitoring, according to the American Society of Anaesthesia (ASA) protocols: five lead ECG, pulse oximetry, non-invasive blood pressure and temperature.

\section{Hemodynamic monitoring:}

- A central line insertion is used for central venous pressure (CVP) monitoring and vasopressor infusion. A second central line/introducer sheath or large-bore peripheral cannula can be used for rapid blood and fluid infusion [7].

- The introducer sheath can be used for pulmonary artery (PA) catheter placement in cases of patients with pulmonary hypertension (PHT) that need constant monitoring.

- Invasive arterial blood pressure measurement represents the gold standard during LT; arterial lines are usually placed after induction.

- One or two arterial lines can be inserted and this varies due to protocols used in different centres.
- One catheter can be inserted in the radial artery; radial artery pressure measurements have the disadvantage of underestimating aortic pressure in hypotensive states when high dose vasopressors are used and after graft reperfusion. More accurate BP measurements can be obtained via a catheter in the femoral/brachial artery. This is the reason why some centres decide to insert a second line in the femoral / brachial artery [8].

CO monitoring: Some centres use pulse contour analysis for stroke volume variation monitoring and fluid guidance. These methods require periodic calibration via thermo-dilution techniques and have the advantage of calculating important parameters of preload $[9,10]$ which can guide fluid management. Insertion of a Swan-Ganz catheter is mandatory in recipients with pulmonary hypertension [11].

Transoesophageal echocardiography (TEE) is not used routinely during liver transplantation but can be an excellent diagnostic tool for potential causes of cardiovascular collapse, such as PHT, hypovolemia, myocardial depression, clot or air embolism. It can also quickly assess the performance of venovenous bypass, contractility, pericardial or pleural effusions. Recipients with recent oesophageal banding are at high risk of gastrointestinal bleeding during TEE insertion and manipulation; it is relatively contraindicated in grade III/IV oesophageal varices [12].

All invasive monitoring should be followed by wellestablished protocols for safety reasons, in order to reduce the risk of hematomas. Ultrasound monitoring is now the gold standard accompanying catheter insertion [13]. The incidence of invasive monitoring complications is low, but femoral site complications, especially infection, can be severe [14].

\section{Neurologic monitoring}

Anaesthetic management and decision making can be guided using different devices and parameters [15].

Most LT centres use bispectral index (BIS) as a guiding tool for anaesthetic depth during both total intravenous anaesthesia (TIVA) and inhalational techniques. Studies have revealed that the use of BIS could decrease intraoperative awareness as it can guide titrate anaesthetic requirements during LT [16].

BIS use is beneficial, especially in acute liver failure (ALF) patients as they often complicate and present with increased intracranial pressure (ICP). During liver transplantation, cerebral oximetry has been used to demonstrate impaired cerebral autoregulation, cerebral 
deoxygenation during the anhepatic phase, and hyperoxygenation after reperfusion of the donor graft.

Jugular venous oxygen saturation (SjvO2) monitoring is another important parameter in ALF patients and shows the balance between oxygen uptake and consumption.

Neuromuscular monitoring is very important for fast track anaesthesia as it can diagnose residual neuromuscular blockade and depressed pharyngeal tonus; it has the great advantage of minimising perioperative complications in LT patients.

Other important parameters need to be monitored constantly during LT. Diuresis must be checked hourly to ensure good renal function, especially because some centres may decide to clamp the vena cava. Constant haemoglobin, haematocrit, electrolytes, base excess and lactate determinations are vital during this procedure as it help guide the anaesthetist in making the best decision for patient management. Viscoelastic testing has become the system of choice for guiding coagulation management and tests are being made according to each patient's bleeding status.

\section{INTRAOPERATIVE ANAESTHESIA}

ESLD patients are at high risk of delayed gastric emptying, and rapid sequence induction (RSI) is usually preferred.

Indications for RSI consist of:

- ascites

- recent food intake

- gastroparesis

Cirrhotic patients present for LT with modified anaesthetic drug pharmacokinetics and pharmacodynamics, which reduce hepatic blood flow and oxygen uptake. They have low albumin levels which increase the bioavailability of many drugs including benzodiazepines; they should be carefully used especially for preoperative anxiolysis as they have a longer half-life, duration of action and reduced protein binding [17].

Induction of general anaesthesia (GA):Intravenous induction with propofol and etomidate are the preferred short-acting intravenous anaesthetic agents for LT recipients

Propofol is frequently used because of its vasodilator effects causing a moderate reduction in hepatic blood flow. Etomidate causes a decrease in hepatic blood flow and has an increased distribution volume with an unpredictable clinical recovery.

Maintenance of GA: The use of a balanced technique with inhalational anaesthetics, non-depolarising muscle relaxants and opioids is recommended.

Cirrhotic patients have reduced inhalational anaesthetic requirements compared to healthy subjects, and this requirement is reflected in the MELD score

Volatile agents decrease mean arterial pressure (MAP) and portal blood flow. Desflurane and sevoflurane have little effect on total hepatic blood flow and are preferred in fast track liver anaesthesia. Fast track anaesthesia can also be achieved with the use of rocuronium (if sugammadex is available), atracurium, remifentanil or fentanyl infusion. Fentanyl is metabolised by the liver, but its elimination is not altered in cirrhotic patients [18].

Due to substantial haemodynamic instability that can suddenly occur, emergency drugs should be available during the three stages of surgery: adrenaline, dopamine and noradrenaline are the drugs of choice. Vasopressin can also be used in case of refractory hypotension as studies showed that ESLD patients have lower vasopressin levels [19].

During a LT procedure, substantial bleeding is almost always anticipated and commonly occurs. It is recommended that cell salvage devices are available for routine use.

The presence of neoplasia or infection represents absolute contraindications for cell salvage devices. Prophylactic antibiotics should be administered before a skin incision is made. but this varies according to each centre; re-dosing is necessary if massive bleeding occurs

Some centres use octreotide in patients with portal hypertension in order to decrease portal pressures and flow during LT. Common side effects of octreotide include: increase in systemic and pulmonary vascular resistance, bradycardia and increase in arterial pressures [20].

\section{Intraoperative fluid management}

Appropriate fluid management is essential during LT. Cirrhotic patients have an abnormal fluid distribution and impaired response to fluid therapy. This is sometimes complicated by bleeding and coagulopathy. Considering all these issues, a thorough assessment of patients' volume status should be regularly performed during the procedure by means of invasive haemodynamic monitoring. 
Excessive fluid therapy can have substantial adverse effects leading, at time, to pulmonary and graft oedema, organ congestion and abnormal gas exchange.

Present studies do not offer a clear answer regarding the ideal monitoring system and fluid therapy in LT patients.

The use of invasive haemodynamic monitoring helps the anaesthetist assess cardiac function and end diastolic volume or preload.

The use of transpulmonary thermodilution techniques offers information about fluid responsiveness with parameters like stroke volume variation (SVV) and global end-diastolic index (GEDI). An important preload parameter is the intrathoracic blood volume (ITBV) which can be used throughout the three stages of LT surgery, especially in the anhepatic phase when the IVC is clamped [21]. Studies show that CVP and pulmonary capillary wedge pressure (PCWP) are not accurate preload parameters [22]. Avoiding plasma transfusion and thus lowering the CVP in the pre-anhepatic phase proved toreduce red blood cell transfusion significantly [23].

Adequate vasoactive substances can offer better hemodynamic stability and organ perfusion, mainly when significant blood loss occurs. Massive transfusion can have substantial adverse effects, and the number of blood products administered intraoperatively can predict patients' readmission to ICU [24]. Goal-directed fluid therapy during LT is crucial as it can decrease post anaesthesia care unit stay (PACU) and mortality [25].

Albumin use proved efficient in reducing the total amount of intraoperative fluid given and reducing the incidence of pulmonary oedema [26]. Studies showed that albumin decreases mortality, reduces the incidence of PRS and the use of vasopressor agents; high costs limit its use. Hetastarch is not recommended because of its side effects: it affects platelet aggregability, decreases the concentration of coagulation factor 8 and increases the risk of bleeding [27]. The use of colloids over crystalloids in liver transplant patients has not been supported by convincing evidence. They can have numerous side effects including anaphylactic reactions, decrease in thrombin generation, worsening of fibrinolysis, specific in the anhepatic phase, and increase the risk of acute kidney injury (AKI) [28].

Crystalloids can also be used during LT. There is no ideal prescription, and all have their limitations:
- Isotonic K crystalloids can be adequate

- Excessive amounts of normal saline (0.9\%) should be avoided as this can lead to hyperchloremic acidosis and a rapid increase in sodium concentrationscausing acute central pontine myelinolysis.

- Ringer lactate (RL): the lactate in RL requires liver metabolism for elimination.

Balanced crystalloid solutions are efficient alternatives for fluid therapy as they have similar plasma electrolytes and osmolarity. One of the significant advantages is that balanced crystalloids contain acetate, which requires extrahepatic metabolism to bicarbonate; some proinflammatory and potential cardiotoxic effects of these solutions have also been described [29].

There is no perfect recipe regarding fluid therapy during LT; a thorough assessment of the patient volemic status and goal-directed fluid management are important measures for a successful procedure.

\section{Pre-anhepatic phase}

This is the first phase of the surgery where liver dissection is carried out. One of the most critical complications during this phase is massive bleeding with a severe impact on the patient's volume status.

It has been shown that surgical experience leads to a significant decrease in bleeding risk during this first phase. However, exceptions can still occur in the presence of high portal hypertension and portosystemic venous shunts, previous surgeries, spontaneous bacterial peritonitis (SBP), and redo LT.

Some LT recipients present with portal hypertension and ascites; its drainage during the procedure can cause hypotension and hemodynamic instability.

Fluid resuscitation is needed to avoid hypovolemia during the second phase when the IVC will be clamped. Albumin $20 \%$ is the preferred option in these patients and RL and $\mathrm{K}$ containing solutions should be avoided.

Cell salvage devices can be used after the evacuation of ascites and before biliary anastomosis. Studies showed that octreotide infusion could be used to reduce portal venous pressures, improve renal function and decrease total red blood cells transfused during LT [30].

One of the disadvantages of bleeding and inappropriate/excessive fluid resuscitation is dilutional coagulopathy and thrombocytopenia, which can be challenging to manage. Studies show that correction of standard coagulation test values may be unreliable and 
inadequate for acute coagulation deficit correction, and viscoelastic testing is recommended for coagulation management during a LT procedure [31].

An advantage of viscoelastic tests is the ability to diagnose hyperfibrinolysis which frequently occurs during liver transplantation, especially in the anhepatic phase. Fortunately, it is self-limited in a good functioning graft. Antifibrinolytic agents can be used when diffuse bleeding occurs or when "point of care tests" indicate hyperfibrinolysis [31].

Anaesthetist should pay particular attention to, and carry out the following during this first stage:

- Maintaining a low CVP can represent an options for reducing bleeding, though the published evidence is conflicting $[32,33]$

- Adequate volume loading is necessary; cirrhotic patients present with marked vasodilation which requires use of vasoactive drugs

- Correct hypothermia and acidosis

- Treat hypocalcaemia

- Maintain $\mathrm{K}$ below $4 \mathrm{mEq} / \mathrm{L}$

- Use "point of care devices" for transfusion guidance

- Treat hyper fibrinolysis and then administer fibrinogen $24 \mathrm{mg} / \mathrm{kg}$ and platelets [34]

- Use of recombinant activated Factor 7

- Veno-venous bypass in case of massive bleeding, hemodynamic instability, complicated surgical procedure, myocardial ischemia, pulmonary hypertension [35]

The anaesthetic goal in this first stage of surgery is to optimise volume status by achieving a balance between fluid perfusion and vasopressors to prepare the patient for the clamping of the IVC in the anhepatic phase.

\section{Anhepatic phase}

It is one of the most challenging parts of the procedure, as the hepatic outflow is obstructed and the IVC can be clamped. These will lead to cardiovascular instability, with a decrease in preload, CO and arterial pressures. Quick diagnosis and management with vasoactive and inotropic drugs are required during this phase of surgery.

Some patients will not tolerate the substantial decrease in preload despite adequate measures. In such cases, hemodynamic collapse can be managed with a surgical portosystemic shunt or a portosystemic venovenous bypass (VVB) where these are available. Some
ESLD patients may present with minimal cardiovascular instability due to a well-developed collateral circulation [36].

During this phase of surgery, the patient is anhepatic, and the total loss of liver function can have the following anaesthetic implications: Patients may become:

- acidotic

- hypocalcaemia due to lack of lactate and citrate metabolism

- hyperkaliaemic

Rapid diagnosis of these critical changes should be made and prompt measures taken to correct them.

Hyperkalaemia and acidemia can be treated with loop diuretics, insulin, glucose, bicarbonate and hyperventilation. Worsening hyperkalaemia, unresponsive to any previous measures, may require intraoperative renal replacement therapy. Tromethamine infusion for $\mathrm{K}$ control has also been suggested in the literature [37].

Due to clamping of the IVC, fluid resuscitation in this phase should be carefully considered and guided by hemodynamic data. Excessive fluid can result in fluid overload during reperfusion which could lead to right heart failure and graft congestion. It has been recommended that adequate arterial pressure is maintained with vasopressors and inotropes and that noradrenaline is the vasopressor of choice during LT [38].

Despite cardiovascular instability, minimal bleeding is normal in the anhepatic phase. Studies have shown that viscoelastic tests represent the gold standard for diagnosis and management of coagulation problems (including hyperfibrinolysis) during LT. Platelets and coagulation factors are decreased due to loss and consumption in the pre-anhepatic phase. Synthesis of coagulation factors and liver clearance do not exist in this phase. The severity of the coagulopathy developed correlates with the length of the anhepatic phase. Hypothermia and acidosis make coagulation problems worse. Coagulopathy develops due to the accumulation of tPA and other anticoagulant factors, including heparinoid products which are generally metabolised by the functioning liver [39]. All these changes cause hyperfibrinolysis and bleeding. No aggressive correction of coagulation abnormalities should be made unless the patient is bleeding since a proper functioning graft will immediately metabolise these products [39].

Some centres prefer using mannitol $(0.5 \mathrm{~g} / \mathrm{kg})$ before IVC clamping in order to avoid liver blood congestion and intraabdominal organs oedema [40]. 
At this stage, the anaesthetist should optimise the patient for graft reperfusion by considering the following:

- Keep serum $\mathrm{K}$ levels below $4 \mathrm{mEq} / \mathrm{L}$

- Calcium levels should be normalised

- Inotropes should be readily available

- There are conflicting studies regarding metabolic acidosis correction. It will self correct with the reperfusion and function of the liver. Minimising blood transfusion helps decrease $\mathrm{K}$ levels and acidosis [41].

In the anhepatic phase, corticoid therapy is frequently started intraoperatively, with a bolus dose of methylprednisolone aceponate (MPA) of 500mg-1g. A common side effect is hyperglycaemia which needs to be monitored regularly during the procedure. Glucocorticoids are usually stopped after one year of treatment [42].

Recipients with hepatitis B receiving hepatitis B negative graft should be infused with hepatitis B hyper immunoglobulin; watch out for allergic reactions.

\section{Neo-hepatic phase}

During the first five minutes after graft reperfusion, significant hemodynamic changes can occur including a sudden decrease in blood pressure, heart rate, SVR and CO. Liver reperfusion can result in worsening of $\mathrm{PHT}$ and right heart failure. A rapid increase in $\mathrm{K}$ is common at this stage and can lead to sinus arrest.

In the absence of VVB, sequestered blood from the portal and lower body venous system suddenly returns to the heart.

Also, high $\mathrm{K}$ preservative solutions and endogenous metabolites accumulated in the liver graft during ischemia time return to the heart. The heart will be overloaded and these products will cause vasodilation, depressed cardiac function and pulmonary vasoconstriction [43]. The typical response of right and left ventricle to stress is abolished due to substances released from the liver in this phase of the surgery [44].

The post reperfusion syndrome (PRS) is defined as a $30 \%$ decrease in the mean arterial pressure for at least a minute and which appears in the first five minutes after graft reperfusion [45]. It can have fatal consequences such as severe arrhythmias or asystole. Dyselectrolytemia, cold solutions used for graft preservation and severe vasodilation due to non-release may contribute to PRS. Studies also show that patients with PRS have an increased risk of postoperative renal dysfunction and fifteen days mortality prediction [46].

Pre-emptive actions before graft reperfusion can be adopted in order to decrease the risk of PRS such as intravenous calcium chloride (1-2g) to stabilise the cardiac membrane, use of inotropes and vasopressors for circulatory support and the administration of bicarbonate and tris (hydroxymethyl) aminomethane (THAM) to correct metabolic acidosis.

Correction of metabolic acidosis with sodium bicarbonate remains controversial. Aggressive treatment of serum $\mathrm{K}$ in the previous stages is vital (K below $4 \mathrm{mEq} / \mathrm{L}$ ). Cell saver usage also helps prevent an increase in $\mathrm{K}$ levels due to massive transfusion. Autologous blood has a low acid content and normal $\mathrm{K}^{+}$concentration, relatively higher2,3-diphosphoglycerate levels and thus provides better cell vitality, preventing complications such as hyperkalaemia [47].

$\mathrm{FiO} 2$ should be maintained at $100 \%$ in order to increase oxygen stores, and inhalational agents should be decreased to minimise vasodilatation [48].

Some studies show the effect of atropine at graft reperfusion before any initial sign of cardiac dysfunction (0.4-1mg). Another option for improving cardiovascular stability is the administration of a bolus of $5 \mathrm{mg}$ ephedrine five minutes before unclamping of the IVC, to maintain a mean arterial pressure (MAP) of 85-100mmHg [49].

In the case of cardiac arrest, direct cardiac massage through a diaphragm incision should be performed. Transoesophageal echocardiography (TEE) is very useful in these severe cases because it can offer a quick diagnosis of cardiac collapse. If intra-cardiac clots are observed, 3000-5000UI heparin bolus via a central line should be administered to prevent clot expansion. Pulmonary thromboembolism can be treated with recombinant tissue plasminogen activators (0.5-4mg)[50].

Bleeding can also occur due to surgical problems or haemostatic abnormalities. Close monitoring of coagulation should be done via viscoelastic testing. No improvement in coagulation observed thirty minutes after graft reperfusion is associated with reduced graft function. Good haemostasis should be achieved before biliary duct anastomosis.

In this stage, the patient needs adequate volemic resuscitation guided by invasive monitoring devices; a significant decrease in vasopressors takes place. 
Anaesthesia for LT is one of the most difficult and challenges anaesthesias. This is due to haemodynamic problems that can arise because of cirrhotic status and also because of the surgical complications during the three stages of surgery. Blood coagulation pattern of the patient has also a major contribution to patients' outcome. A good understanding of the recipient's pathophysiology and an adequate perioperative management are decisive for good LT outcome.

\section{CONCLICT OF INTEREST}

None to declare.

\section{REFERENCES}

1. Tovikkai C, Charman SC, Praseedom RK, Gimson AE, Van der Meulen J. Time-varying impact of comorbidities on mortality after liver transplantation: a national cohort study using linked clinical and administrative data. BMJ open. 2015;5(5):e006971

2. Jimenez-Romero C, Caso Maestro O, CambraMolero F, et al. Using older liver grafts for liver transplantation: where are the limits?. World J Gastroenterol. 2014;20(31):10691-10702

3. Graziadei I, Zoller H, Fickert P, et al. Indications for liver transplantation in adults: recommendations of the Austrian Society for Gastroenterology and Hepatology in cooperation with the Austrian Society for Transplantation, Transfusion and Genetics. Wien KlinWochenschr. 2016;128(19-20):679-690

4. Massie AB, Caffo B, Gentry SE, et al. MELD Exceptions and Rates of Waiting List Outcomes. Am J Transplant. 2011;11(11):23622371

5. Bernal W, Williams R. Beyond $\mathrm{KCH}$ selection and options in acute liver failure. Hepatol Int. 2018;12(3):204-213

6. Zhu Z. Milan criteria and its expansions in liver transplantation for hepatocellular carcinoma. Hepatobiliary surgery and nutrition.2016.5(6):498-502.

7. Singh A, Nasa V, Tandon M. Perioperative monitoring in liver transplant patients. J Clin Exp Hepatol. 2012;2(3):271-278

8. Schumann R, Mandell MS, Mercaldo N, et al. Anesthesia for liver transplantation in United States academic centers: intraoperative practice. J Clin Anesth. 2013;25(7):542-550.

9. Biancofore G, Critchley LA, Lee $A$, et al. Evaluation of an uncalibrated arterial pulse contour cardiac output monitoring system in cirrhotic patients undergoing liver surgery. $\mathrm{Br} \mathrm{J}$ Anaesth. 2009;102(1):47-54

10. Rudnick MR, Marchi LD, Plotkin JS. Hemodynamic monitoring during liver transplantation: A state of the art review. World J Hepatol. 2015;7(10):1302-1311

11. Bias M, Nouette-Gaulain K, Cottenceau V, et al. Cardiac output measurement in patients undergoing liver transplantation: pulmonary artery catheter versus uncalibrated arterial pressure waveform analysis. AnesthAnalg. 2008;106(5):14801486

12. De Pietri L, Mocchegiani F, Leuzzi C, et al. Transoesophageal echocardiography during liver transplantation. World J Hepatol. 2015;7(23):2432-2448

13. Lu SY, Matsusaki T, Abuelkasem E, et al. Complications related to invasive hemodynamic monitors during adult liver transplantation. Clin Transplant. 2013;27(6):823-828

14. Kim SK, Shin WJ, Kim JW, Park JY, Hwang GS. Prediction of hyperdynamic circulation by arterial diastolic reflected waveform analysis in patients undergoing liver transplantation. Blood Press Monit. 2016;21(1):9-15

15. Mendizabal M, Silva OM. Liver transplantation in acute liver failure: a challenging scenario. World J Gastroenterol. 2016;22(4):1523-1531

16. Cao YH, Chi P, Zhao YX, Dong XC. Effect of bispectral indexguided anesthesia on consumption of anesthetics and early postoperative cognitive dysfunction after liver transplantation. Medicine (Baltimore). 2017;96(35):e7966

17. Rahimzadeh P, Safari S, Faiz SH, Alavian SM. Anesthesia for patients with liver disease. Hepat Mon. 2014;14(7):e19881

18. Aniskevich S, Pai SL. Fast track anesthesia for liver transplantation: review of the current practice. World J Hepatol. 2015;7(20):2303-2308

19. Wagener G, Kovalevskaya G, Minhaz M, Mattis F, Emond JF, Landry DW. Vasopressin deficiency and vasodilatory state in end-stage liver disease. J CardiothoracVascAnesth. 2011;25(4):665-670

20. Busani S, Marconi G, Schiavon L, et al. Living donor liver transplantation and management of portal venous pressure. Transplant Proc. 2006;38(4):1074-1075

21. Della Rocca G, Brondani A, Costa MG. Intraoperative hemodynamic monitoring during organ transplantation: What is new?. CurrOpin Organ Transplant.2009;14(3):291-296

22. Lucey MR. Liver transplantation in patients with alcoholic liver disease. Liver Transpl.2011;17(7):751-759

23. Gorlinger K. Coagulation management during liver transplantation. Hämostaseologie. 2006;26(3 Suppl 1):S64-76

24. Levy MF, Greene L, Ramsay MA, et al. Readmission to the intensive care unit after liver transplantation. Crit Care Med. 2001;29(1):18-24

25. Marik PE. Noninvasive cardiac output monitors: A state of the art review. J CardiothoracVascAnesth. 2013;27(1):121-134

26. Haynes GR, Navickis RJ, Wilkes MM. Albumin administrationwhat is the evidence of clinical benefit? A systematic review of randomized controlled trials. Eur J Anaesthesiol. 2003;20(10):771-793

27. Hand WR, Whiteley JR, Epperson TI, et al. Hydroxyethyl starch and acute kidney injury in orthotopic liver transplantation: a single-center retrospective review. AnesthAnalg. 2015;120(3):619-626

28. Groeneveld AB,Navickis RJ, Wilkes MM. Update on the 
Available online at: www.jccm.ro

comparative safety of colloids: a systematic review of clinical studies. Ann Surg. 2011;253(3):470-483

29. Nandhakumar A, McCluskey SA, Srinivas C, Chandy TT. Liver transplantation: advances and perioperative care. Indian J Anaesth. 2012.56(4):326-335

30. Sahmeddini MA, Amini A, Naderi N. The effect of octreotide on urine output during orthotopic liver transplantation and early postoperative renal function; a randomized, double-blind, placebo-controlled trial. Hepat Mon. 2013;13(9):e12787

31. Mallett SV. Clinical utility of viscoelastic tests of coagulation (TEG/ROTEM) in patients with liver disease and during liver transplantation. SeminThrombHemost. 2015;41(5):527-537

32. Lutz JT, Valentin-Gamazo C, Gorlinger K, Malago M, Peters J. Blood-transfusion requirements and blood salvage in donors undergoing right hepatectomy for living related liver transplantation. AnesthAnalg. 2003;96(2):351-355

33. Feng $Z Y, X u X, Z$, hu SM, Bein $B$, Zheng SS. Effects of low central venous pressure during preanhepatic phase on blood loss and liver and renal function in liver transplantation. World J Surg. 2010;34(8):1864-1873

34. Donohue $\mathrm{Cl}$, Mallett SV. Reducing transfusion requirements in liver transplantation. World J Transplant. 2015;5(4):165-182

35. Fonouni $H$, Mehrabi A, Soleimani M, Müller SA, Büchler $\mathrm{MW}$, Schmidt J. The need for venovenous bypass in liver transplantation. HPB (Oxford). 2008;10(3):196-203

36. Sharma M, Rameshbabu CS. Collateral pathways in portal hypertension. Journal of clinical and experimental hepatology. 2012.2(4), 338-352.

37. Adelmann D, Kronish K, Ramsay MA. Anesthesia for liver transplantation. Anesthesiol Clin. 2017;35(3):491-508

38. Dalal A. Anesthesia for liver transplantation. Transplant Rev (Orlando). 2016;30(1):51-60

39. Senzolo M, Cholongitas E, Thalheimer U, et al. Heparin-like effect in liver disease and liver transplantation. Clin Liver Dis. 2009;13(1):43-53

40. Vater Y, Levy A, Martay K, Hunter C, Weinbroum AA. Adjuvant drugs for end-stage liver failure and transplantation. Med Sci
The Journal of Critical Care Medicine 2020;6(2) • 99

Monit. 2004;10:RA77-88

41. Weinberg L, Broad J, Pillai P, et al. Sodium bicarbonate infusion in patients undergoing orthotopic liver transplantation: a single center randomized controlled pilot trial. Clin Transplant. 2016;30(5):556-565

42. Moini M, Schilsky ML, Tichy EM. Review of immunosuppression in liver transplantation. World J Hepatol. 2015;7(10):13551368

43. Cao Z, Gao Y, Tao G. Vasoplegic syndrome during liver transplantation. AnesthAnalg. 2009;108(6):1941-1943

44. Kashimutt S, Kotze A. Anaesthesia for liver transplantation. BJA Education. 2017;17(1):35-40

45. Fukazawa K, Pretto EA. The post-reperfusion syndrome (PRS): Diagnosis, incidence and management. In Liver Transplantation-Basic Issues. 2012 Available from http:// intechopen.com/books/liver-transplantation-basicissues/ the-post-reperfusionsyndrome-prs-diagnosis-incidence-andmanagement

46. Bukowicka B, Akar RA, Olszewska A, Smoter P, Krawczyk M. The occurrence of postreperfusion syndrome in orthotopic liver transplantation and its significance in terms of complications and short-term survival. Ann Transplant. 2011;16(2):26-30

47. Biddy MF, Ridler B, Thompson JF. The qualities of blood reinfused during cell salvage. Transfusion Alternatives in Transfusion Medicine. 2003;5(5):466-471

48. Valentine E, Gregorits M, Gutsche JT, Al-Ghofaily L, Augoustides JG. Clinical update in liver transplantation. J CardiothoracVascAnesth. 2013;27(4):809-815

49. Fayed NA, Murad WS. Goal directed preemptive ephedrine attenuates the reperfusion syndrome during adult living donor liver transplantation. Egyptian Journal of Anaesthesia. 2014;30:187-195

50. Boone JD, Sherwani SS, Herborn JC, Patel KM, De Wolf AM. The successful use of low-dose recombinant tissue plasminogen activator for treatment of intracardiac/pulmonary thrombosis during liver transplantation. AnesthAnalg. 2011;112(2):319321 